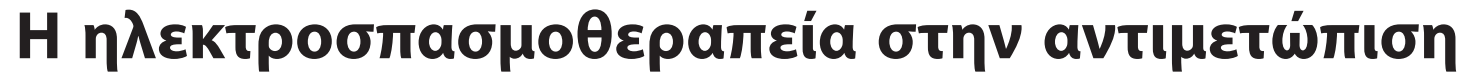

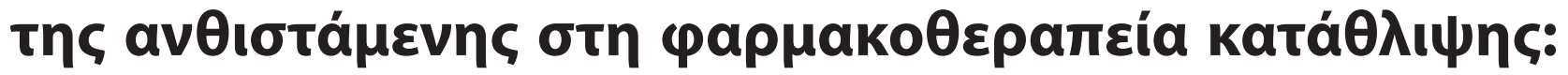

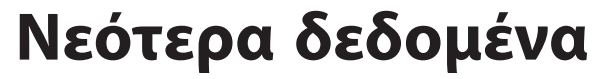

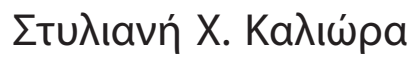

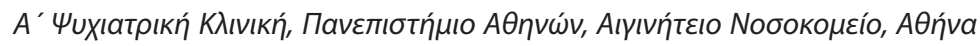

\begin{abstract}
ПЕРІАНЧН

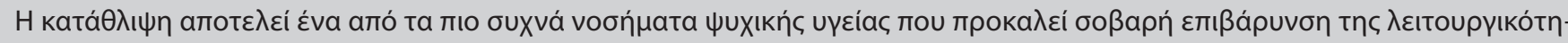

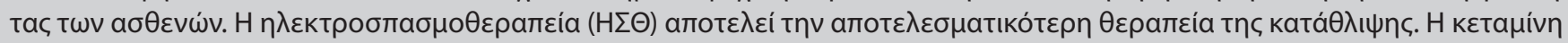

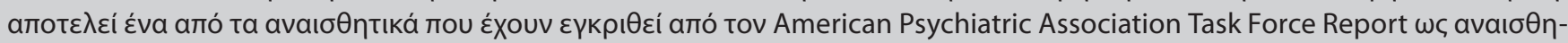

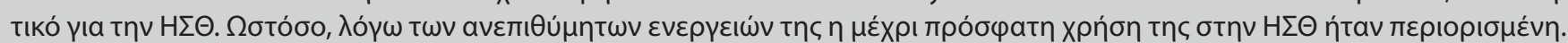

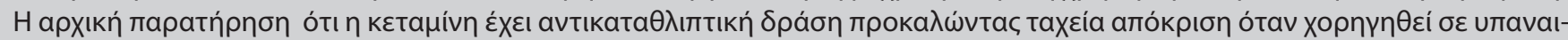

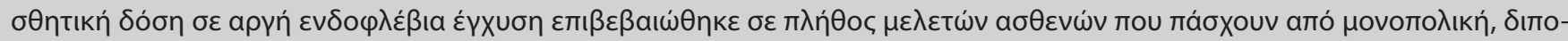

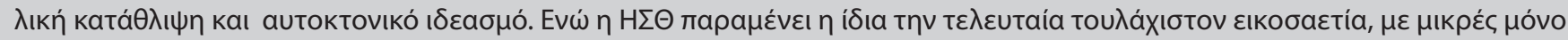

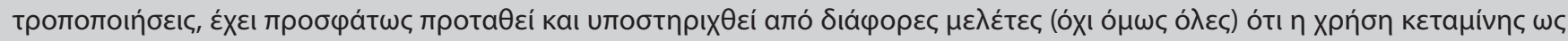

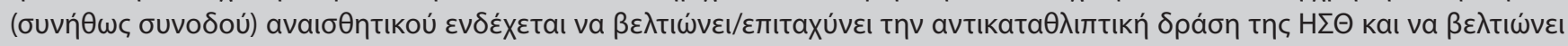

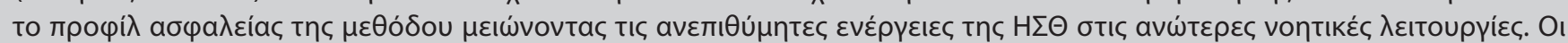

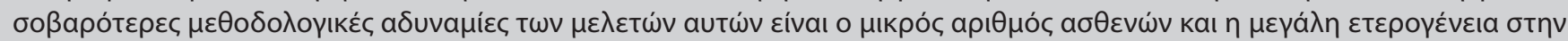

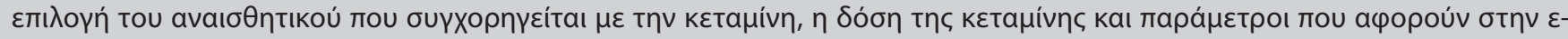

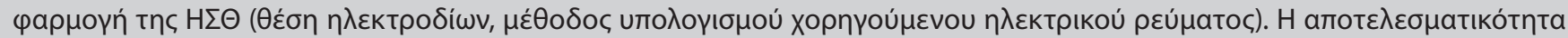

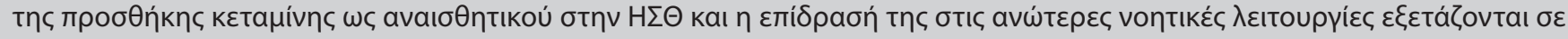

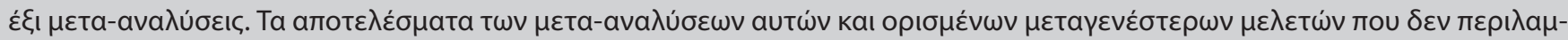

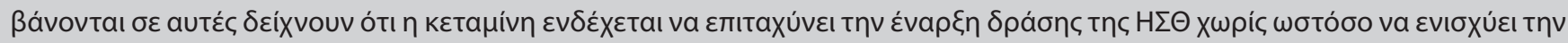

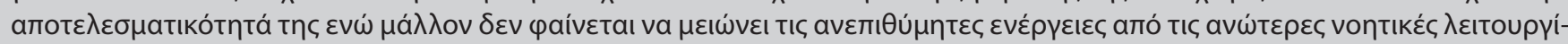

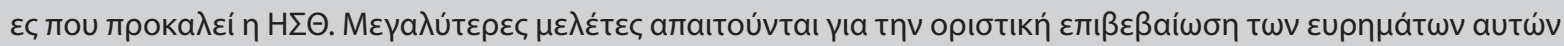

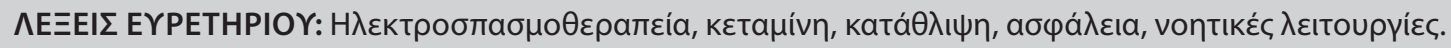

\section{Eıoapwpń}

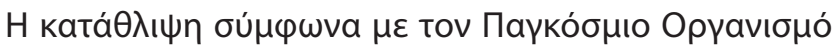

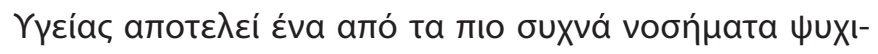

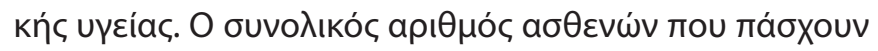

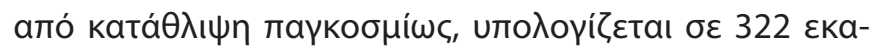

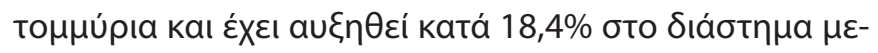

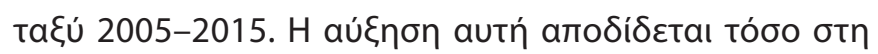

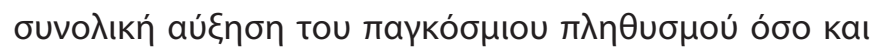

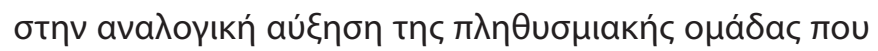

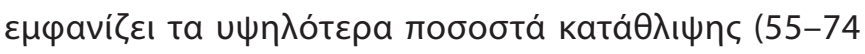

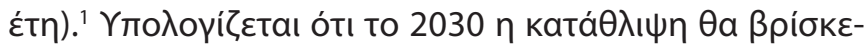

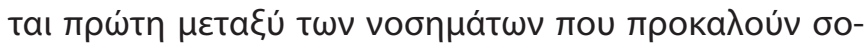

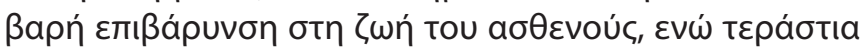

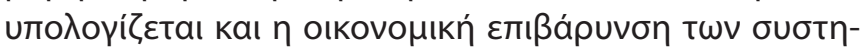

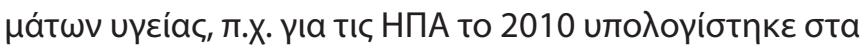

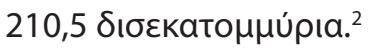

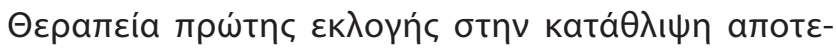

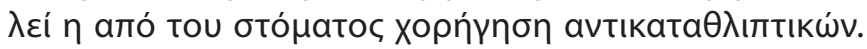

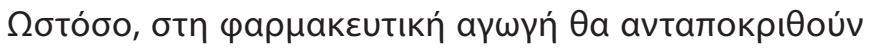




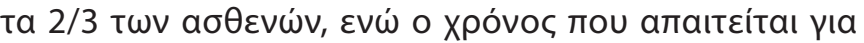

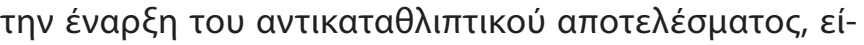

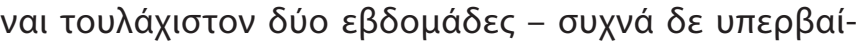

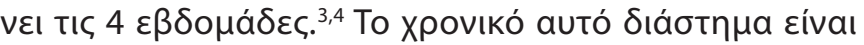

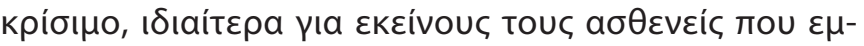

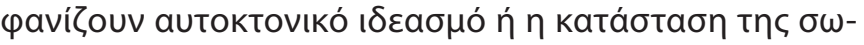

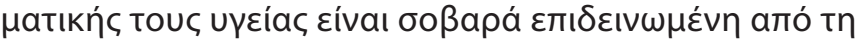

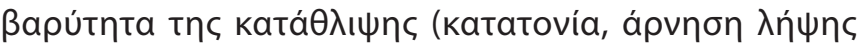

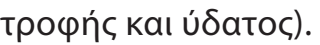

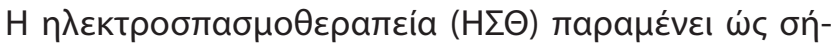

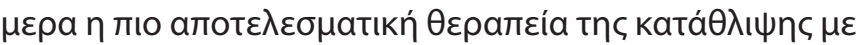

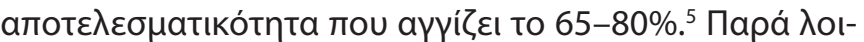

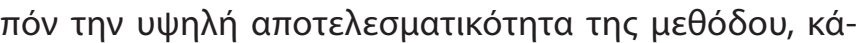

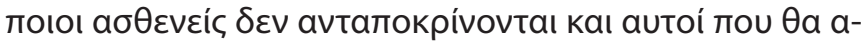

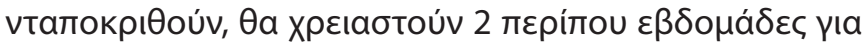

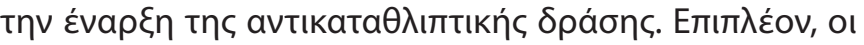

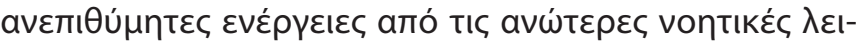

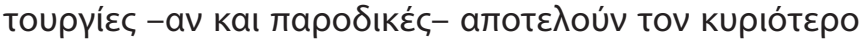

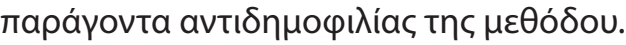

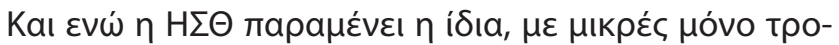

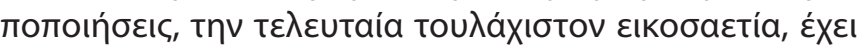

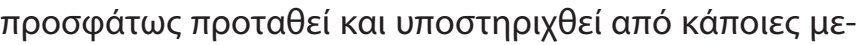

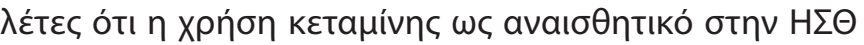

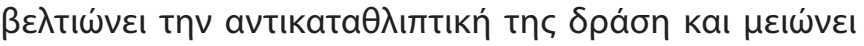

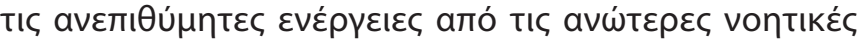

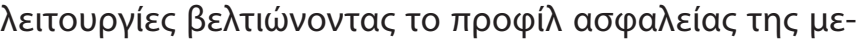

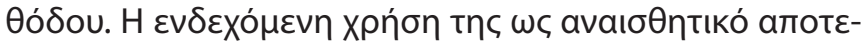

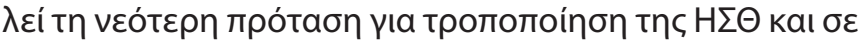

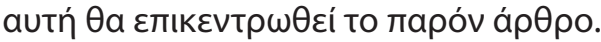

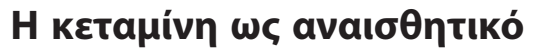

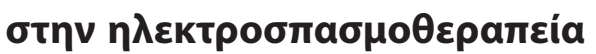

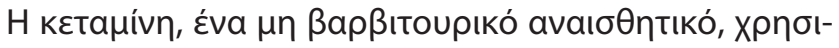

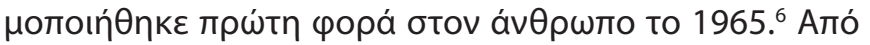

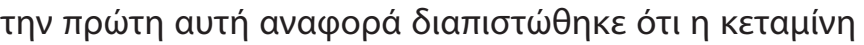

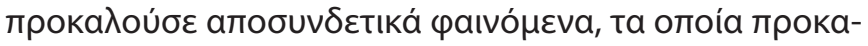

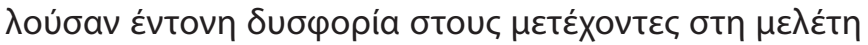

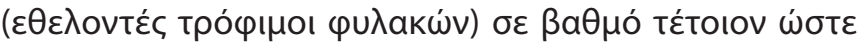

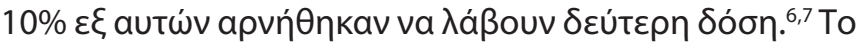

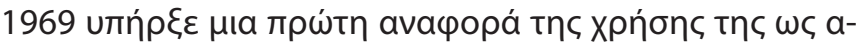

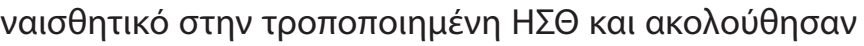

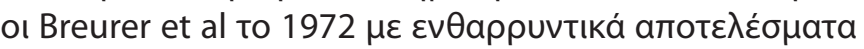

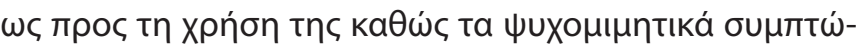

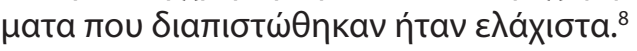

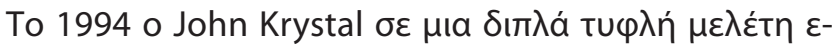

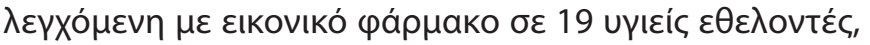

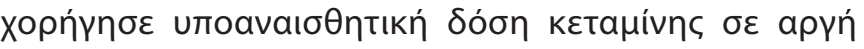

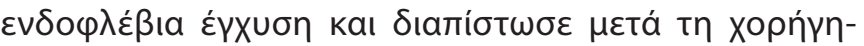

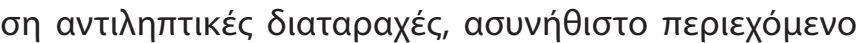

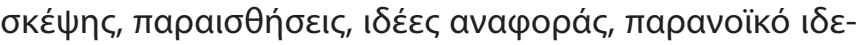

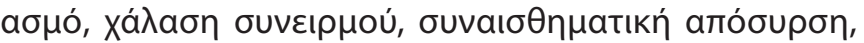

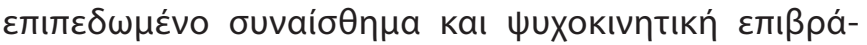

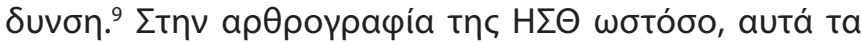

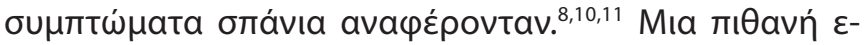

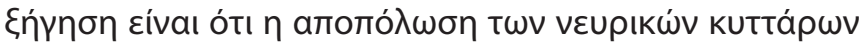

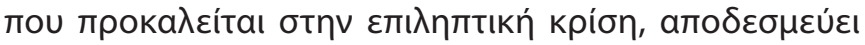

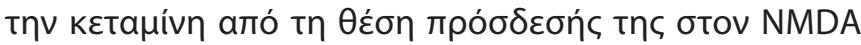

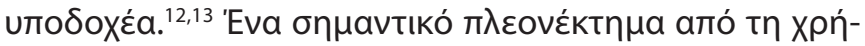

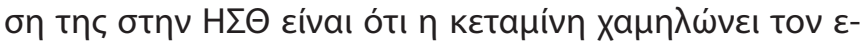

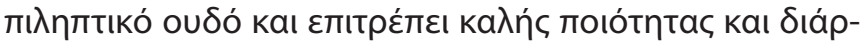

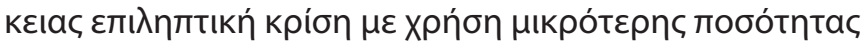

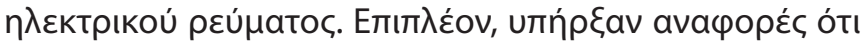

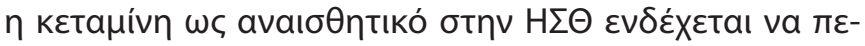

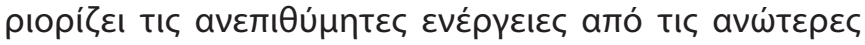

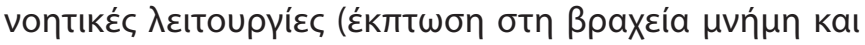

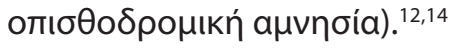

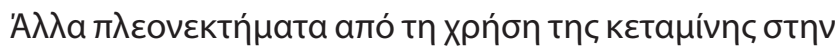

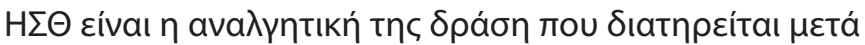

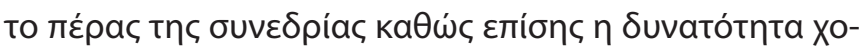

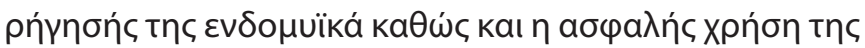

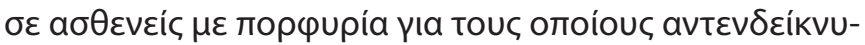

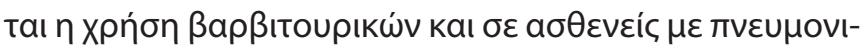

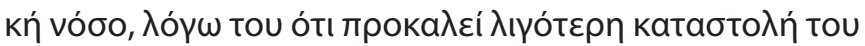

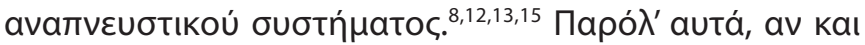

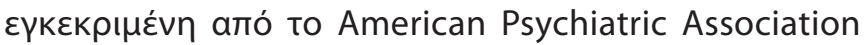

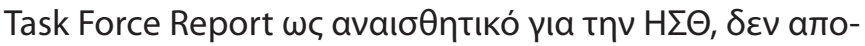

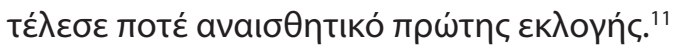

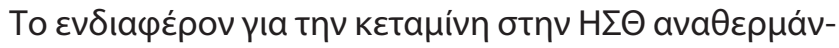

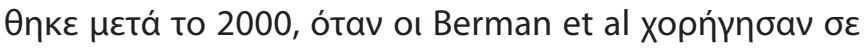

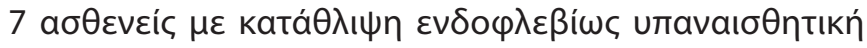

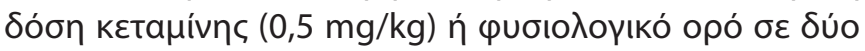

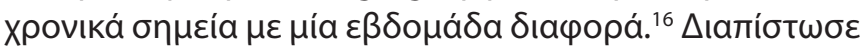

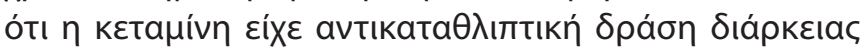

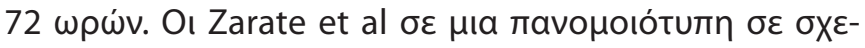

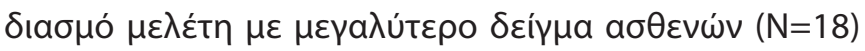

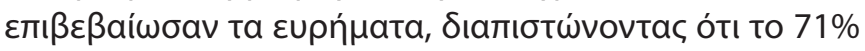

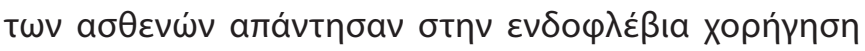

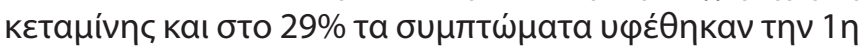

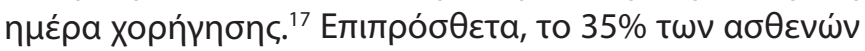

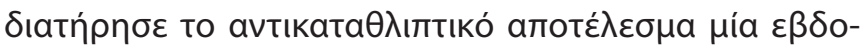

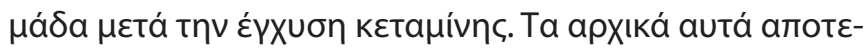

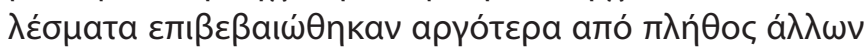

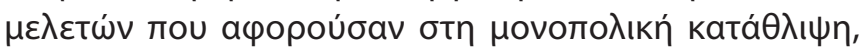

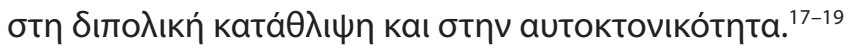

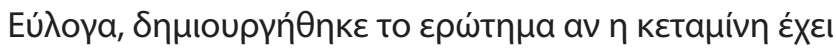

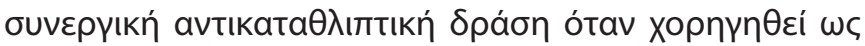

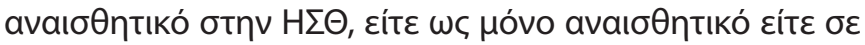

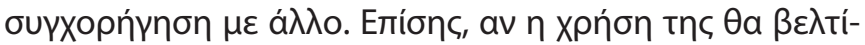

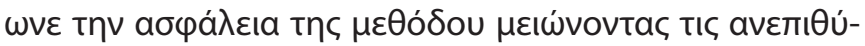

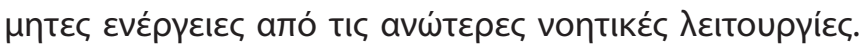

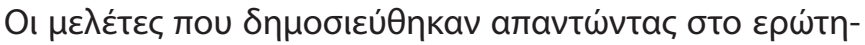

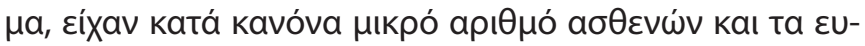

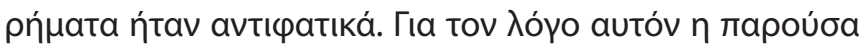

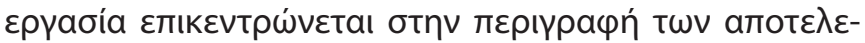




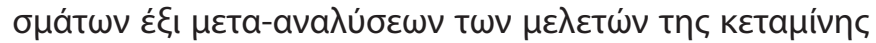

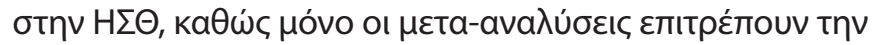

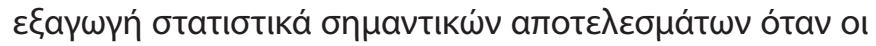

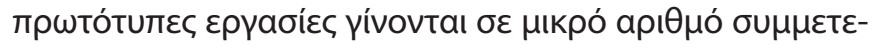

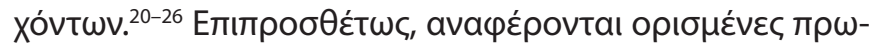

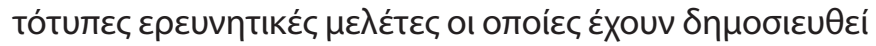

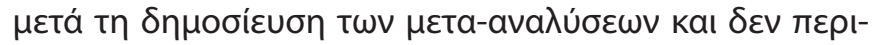
$\lambda a \mu \beta a ́ v o v t a ı ~ \sigma \varepsilon$ autéc.

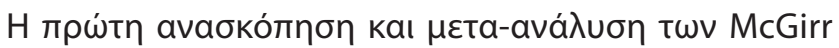

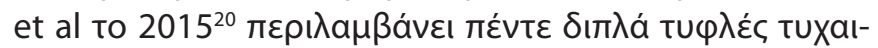

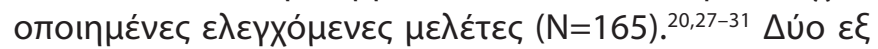

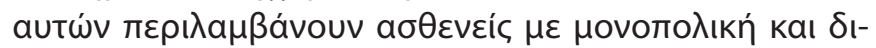

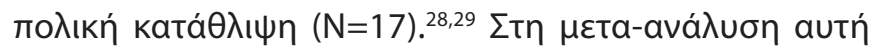

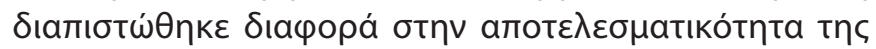

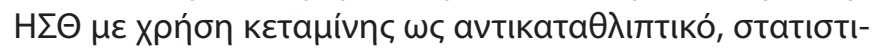

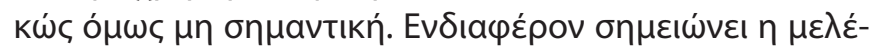

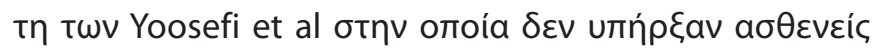

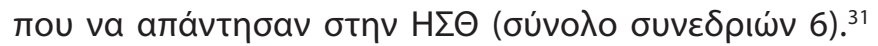

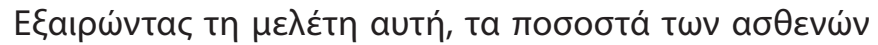

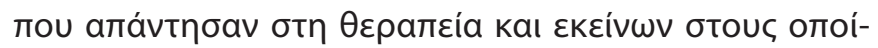

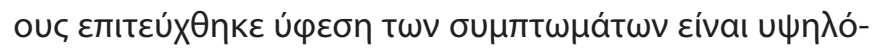

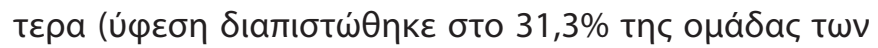

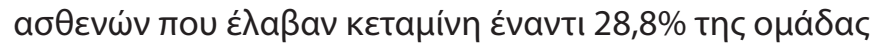

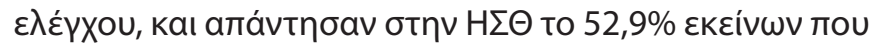

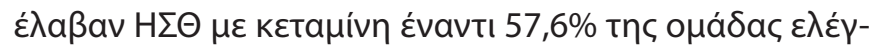

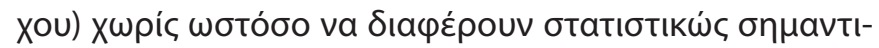
кá $\mu \varepsilon t a \xi u ́$ touc.

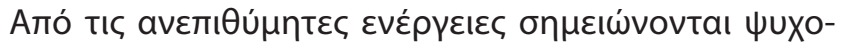

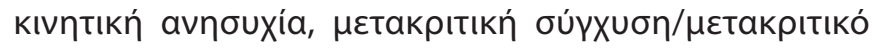

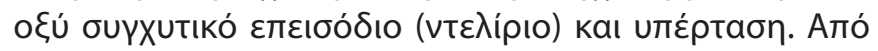

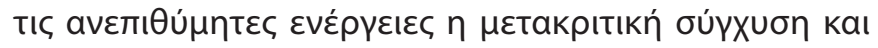

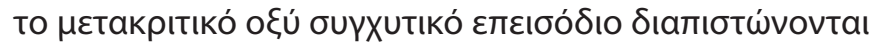

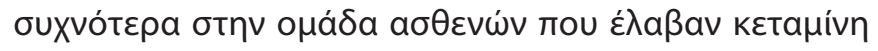

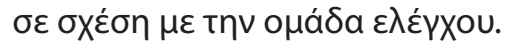

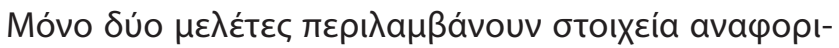

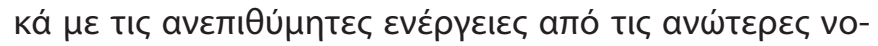

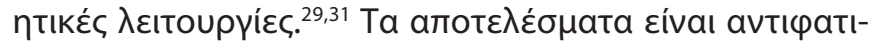

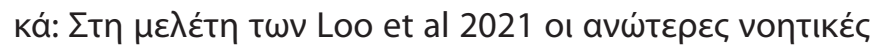

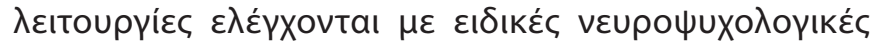

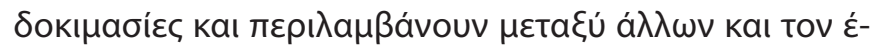

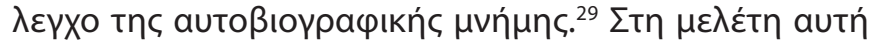

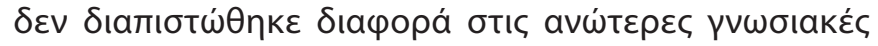

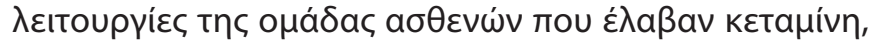

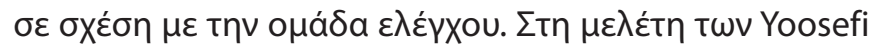

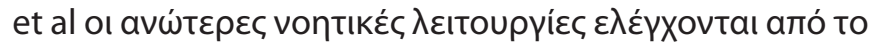

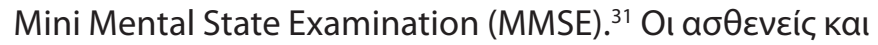

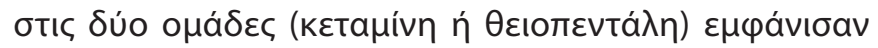

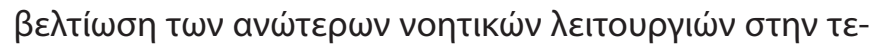

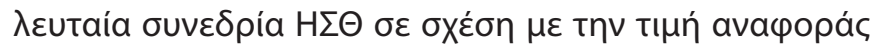

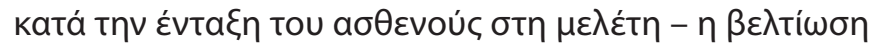

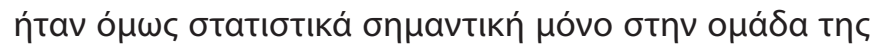

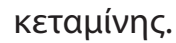

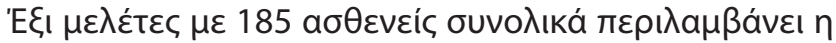

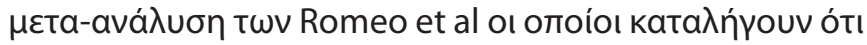

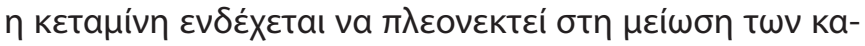
таӨ

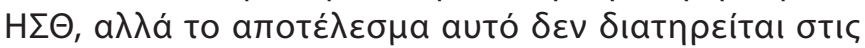

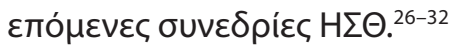

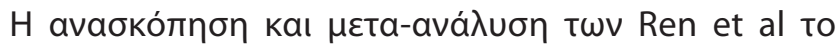

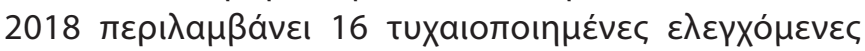

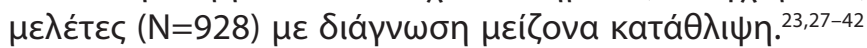

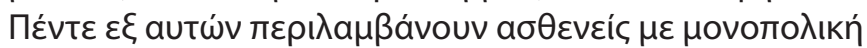

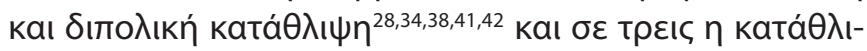

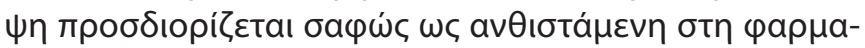

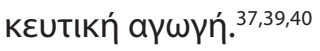

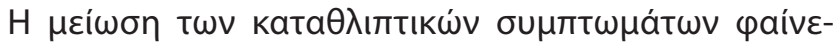

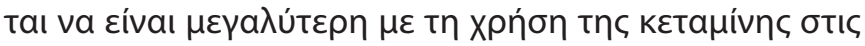

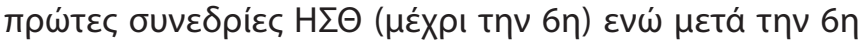

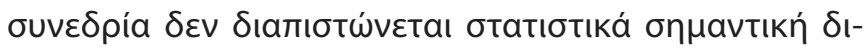

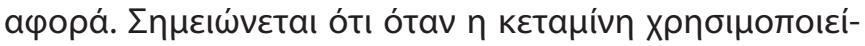

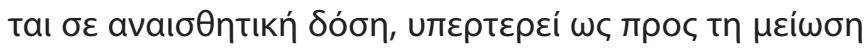

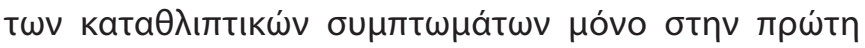

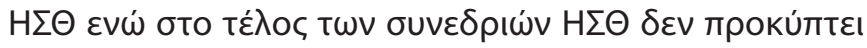

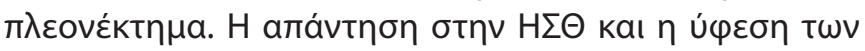

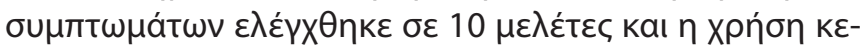

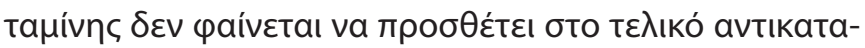

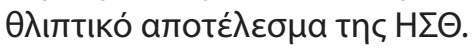

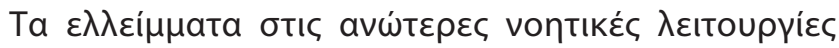

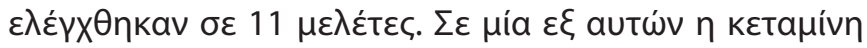

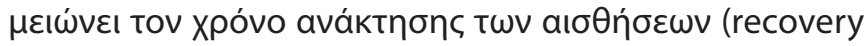

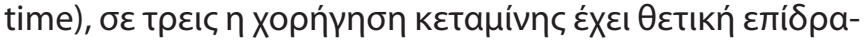

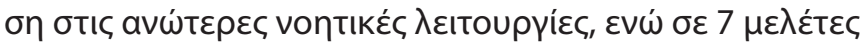

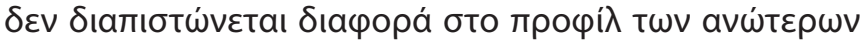

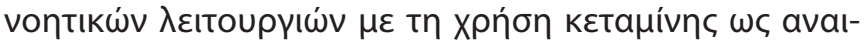

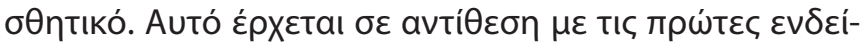

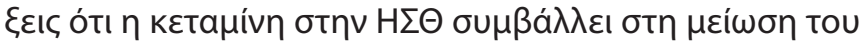

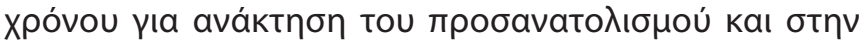

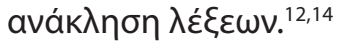

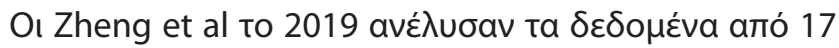

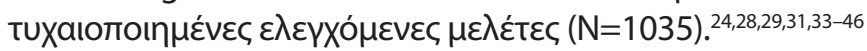

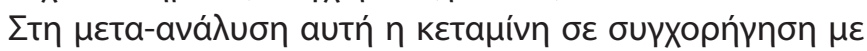

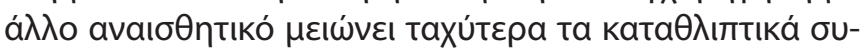

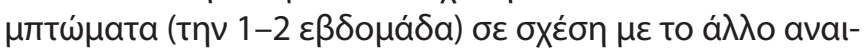

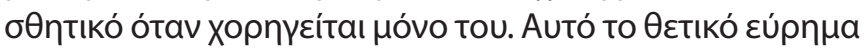

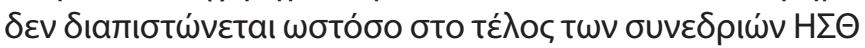

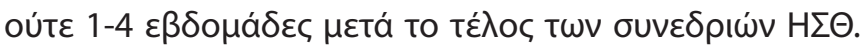

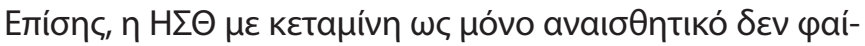

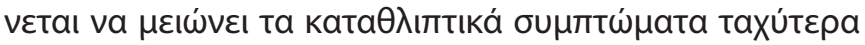

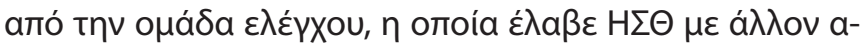

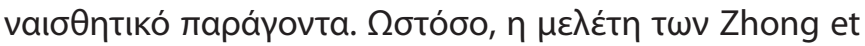

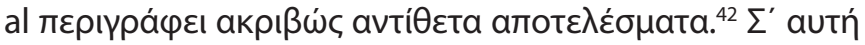

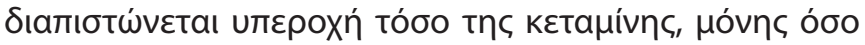

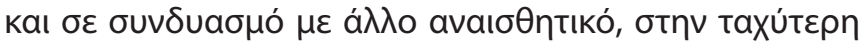

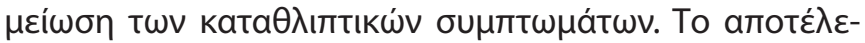




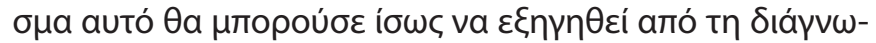

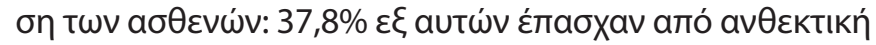

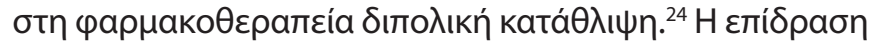

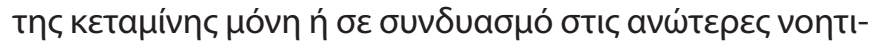

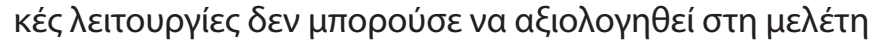

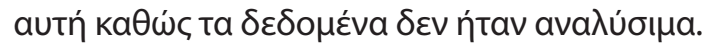

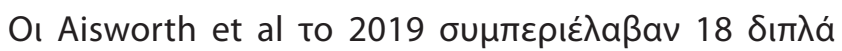

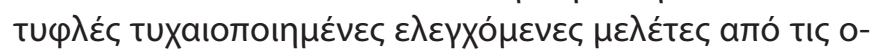

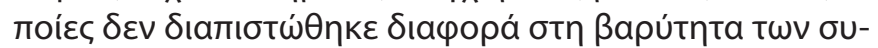

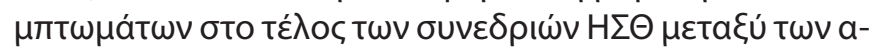

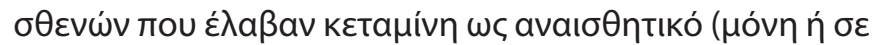

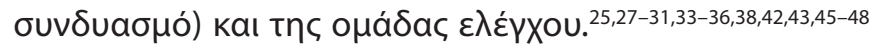

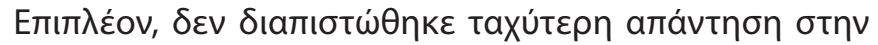

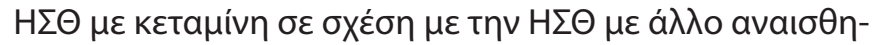
TIKó.

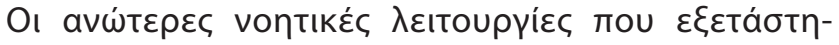

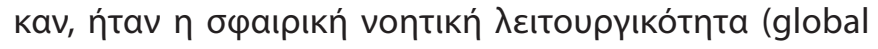

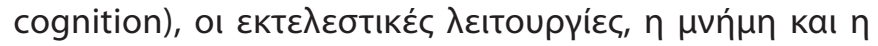

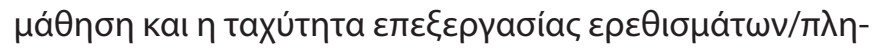

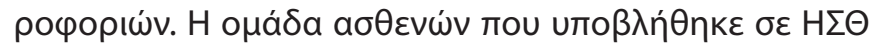

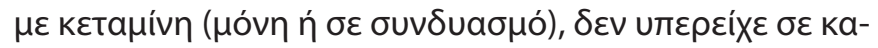

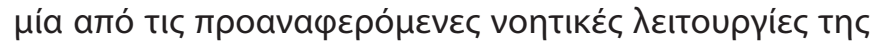

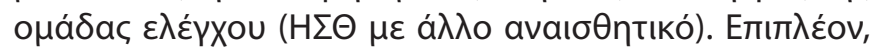

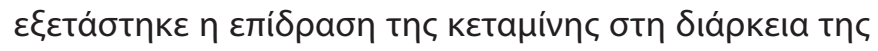

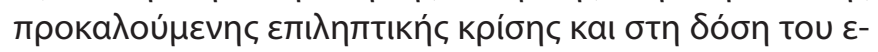

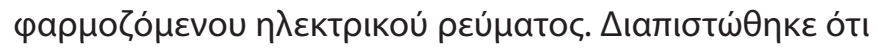

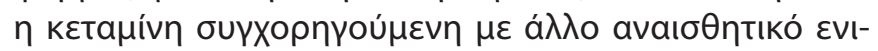

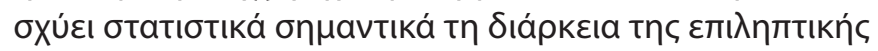

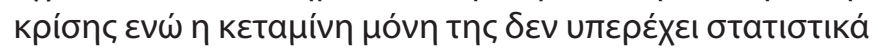

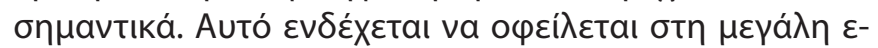

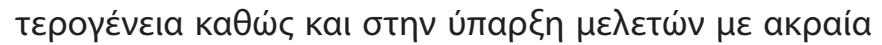

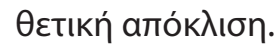

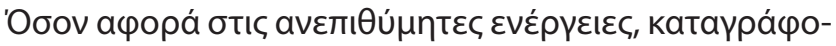

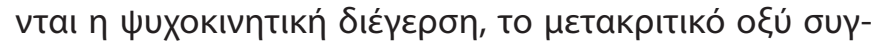

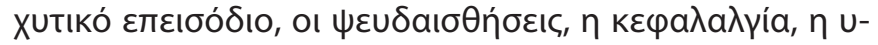

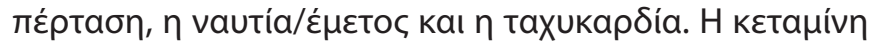

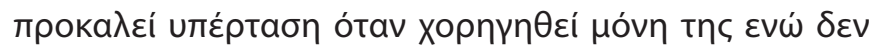

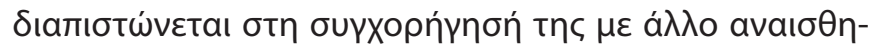

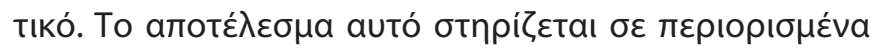

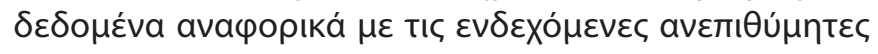

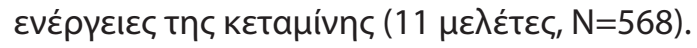

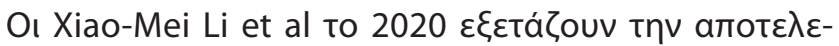

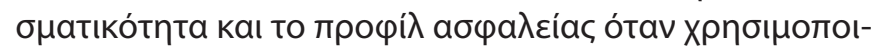

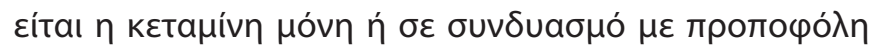

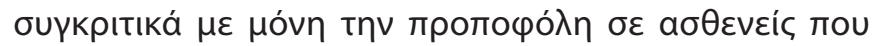

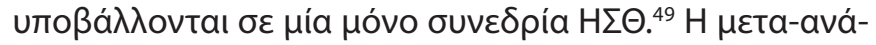

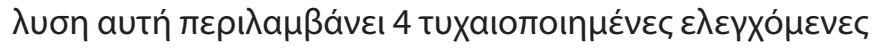

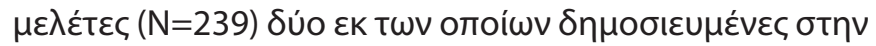

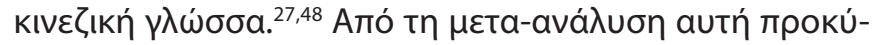

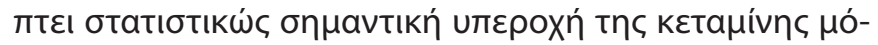

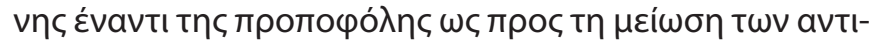

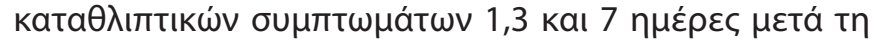

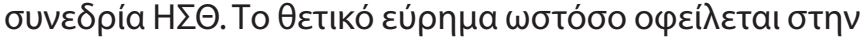

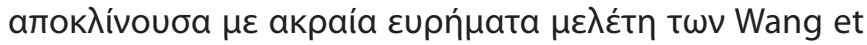

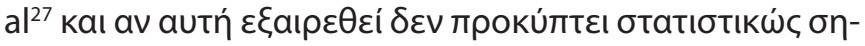

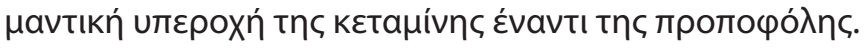

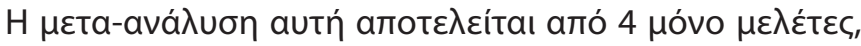

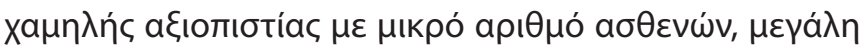

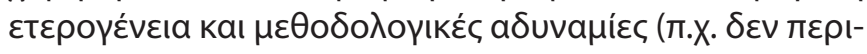

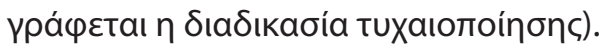

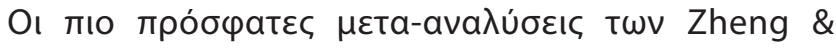

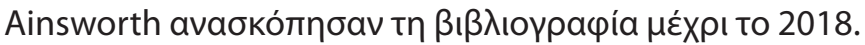

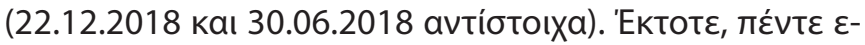

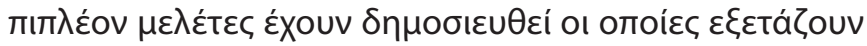

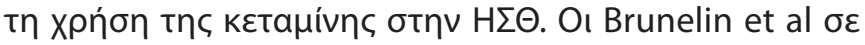

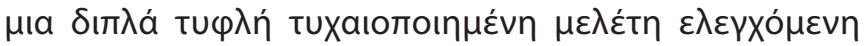

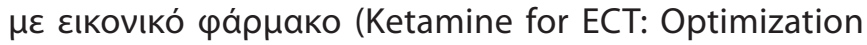

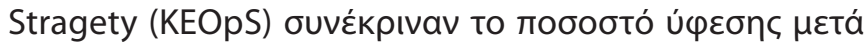

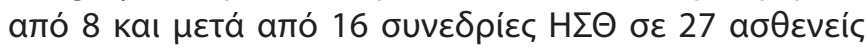

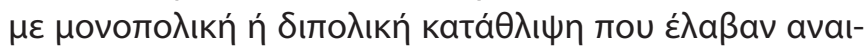

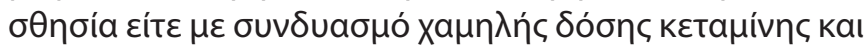

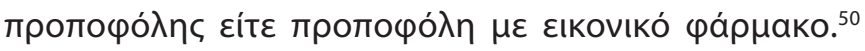

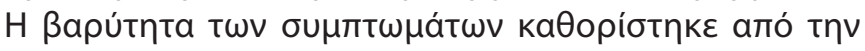

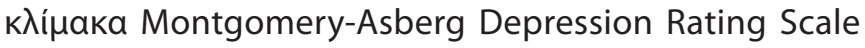

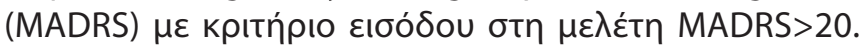

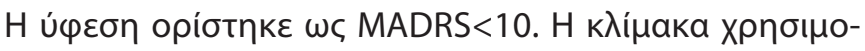

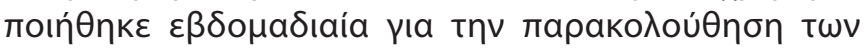

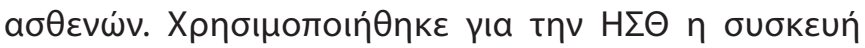
Spectrum 5000Q (Mecta Corporation, TuaLatin, OR, USA)

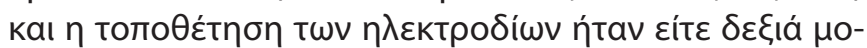

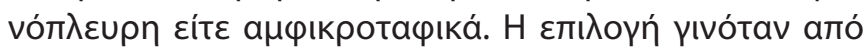

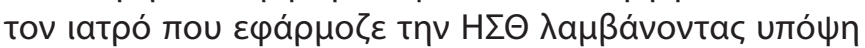

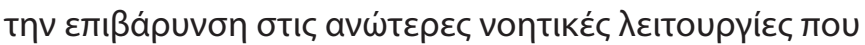

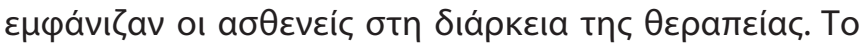

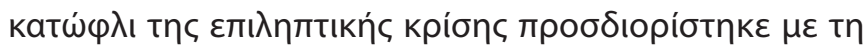

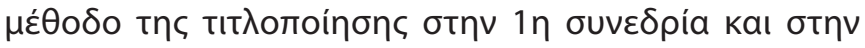

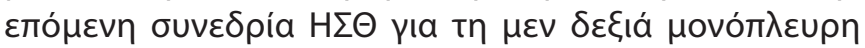

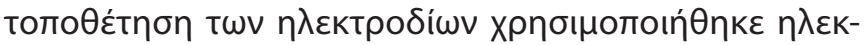

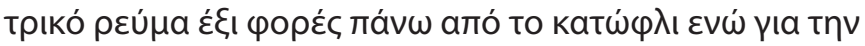

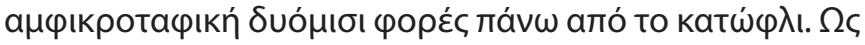

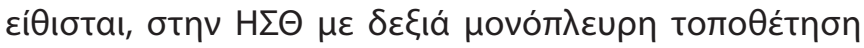

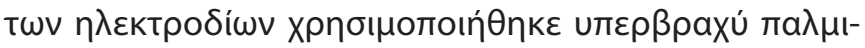

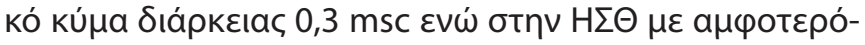

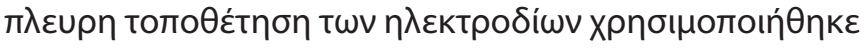

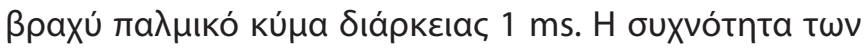

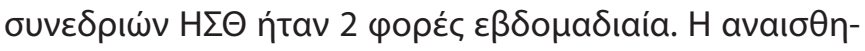

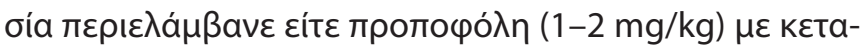

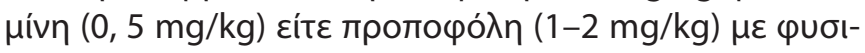
о入оүıкó орó.

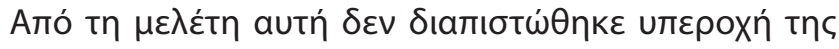

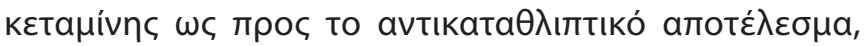

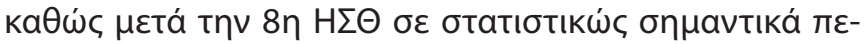

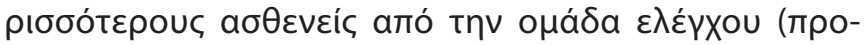

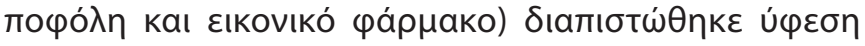

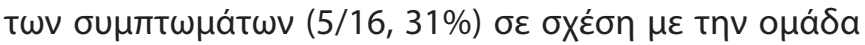




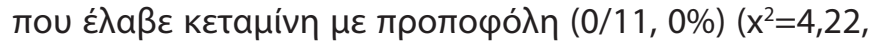

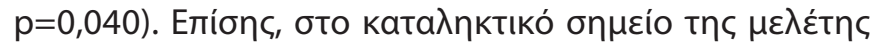

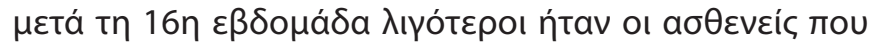

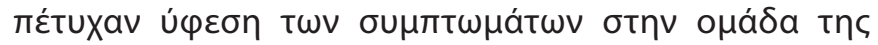

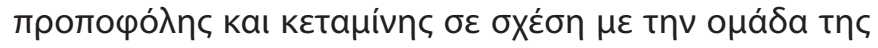

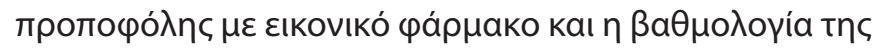

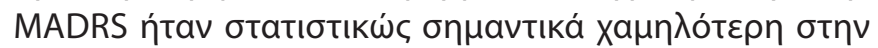

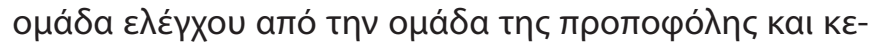

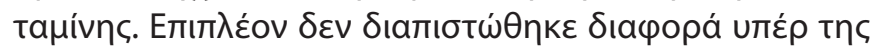

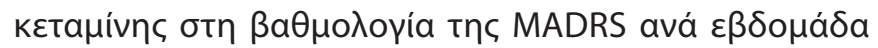

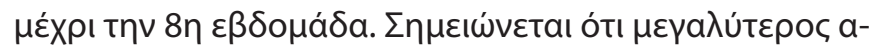

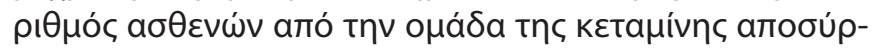

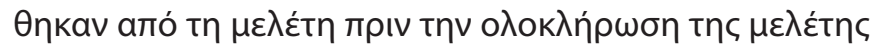

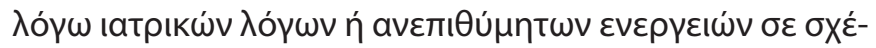

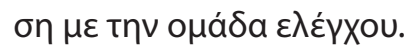

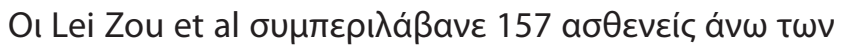

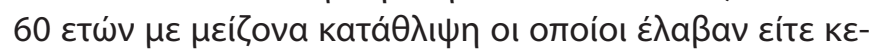

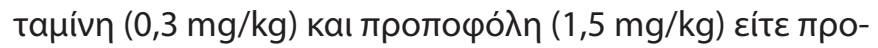

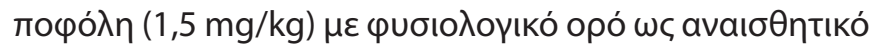

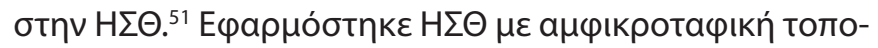

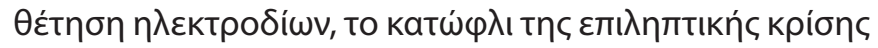

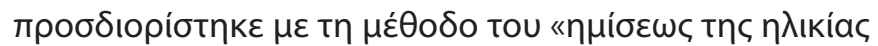

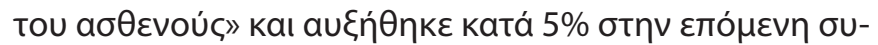

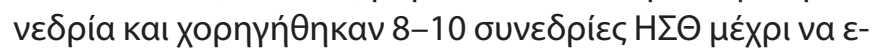

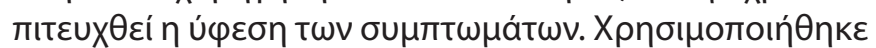

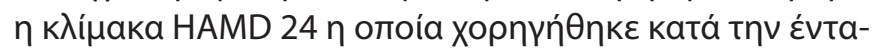

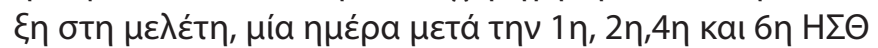

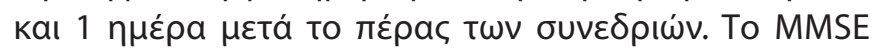

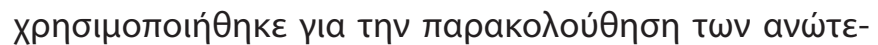

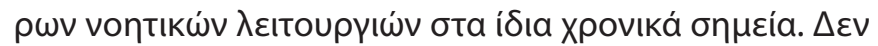

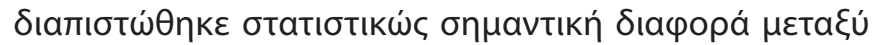

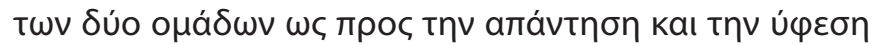

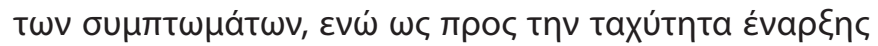

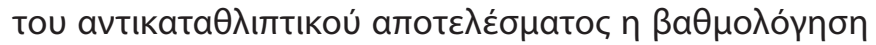

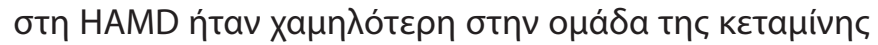

\section{BıBntoypacpía}

1. World Health Organization. Depression and Other Common Mental Disorders: Global Health Estimates. World Health Organization, Geneva, 2017. Available at http://appswhoint/iris

2. Greenberg PE, Fournier AA, Sisitsky T, Pike CT, Kessler RC. The economic burden of adults with major depressive disorder in the United States (2005 and 2010). J Clin Psychiatry 2015, 76:155-162, doi: 10.4088/ JCP.14m09298

3. Gelenberg AJ, Chesen CL. How fast are antidepressants? J Clin Psychiatry 2000, 61:712-721, doi: 10.4088/jcp.v61n1002

4. Quitkin FM, Rabkin JG, Ross D, Stewart JW. Identification of true drug response to antidepressants. Use of pattern analysis. Arch Gen Psychiatry 1984, 41:782-786, doi: 10.1001/archpsyc.1984.01790 190056007

5. Petrides G, Fink M, Husain MM, Knapp RG, Rush AJ, Mueller M et al. ECT remission rates in psychotic versus nonpsychotic depressed patients:

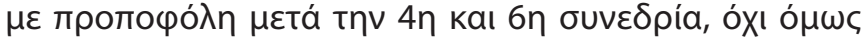

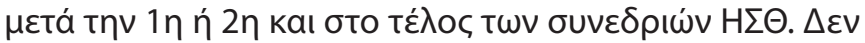

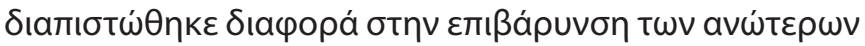

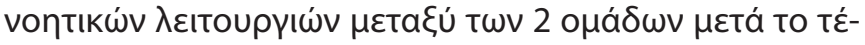

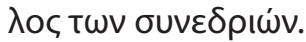

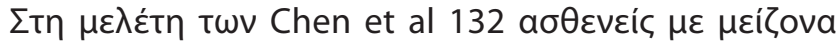

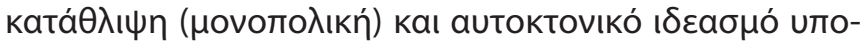

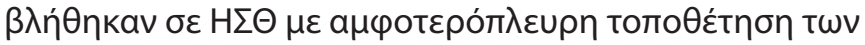

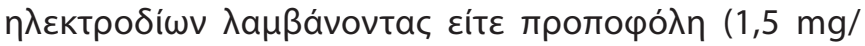

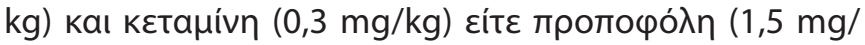

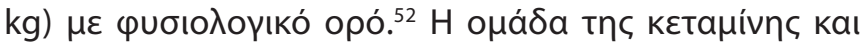

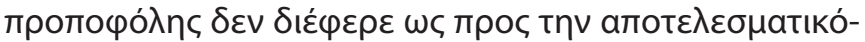

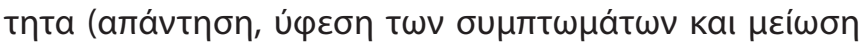

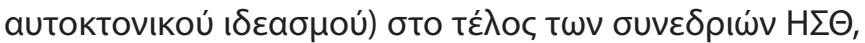

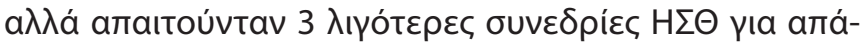

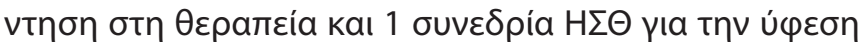

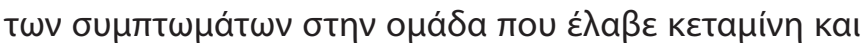

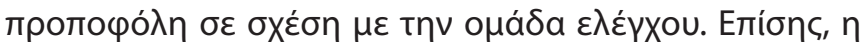

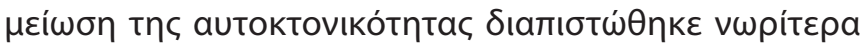

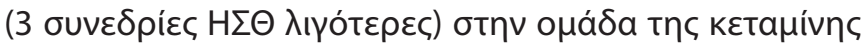

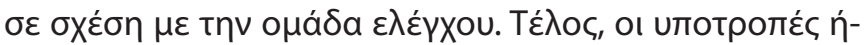

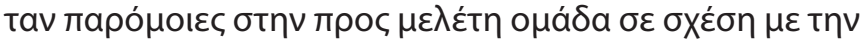

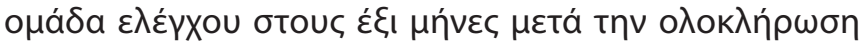

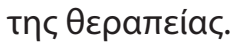

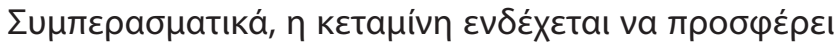

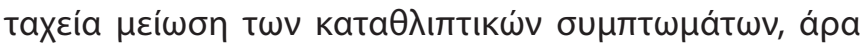

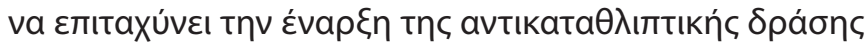

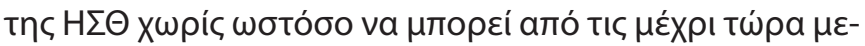

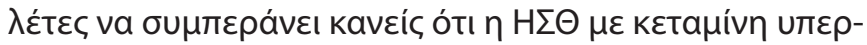

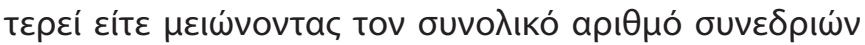

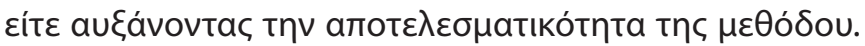

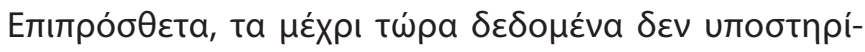

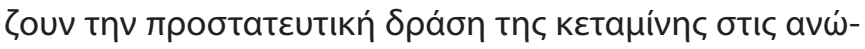

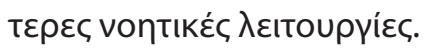

a report from CORE. J ECT 2001, 17:244-253, doi: 10.1097/00124509200112000-00003

6. Domino EF, Chodoff P, Corssen G. Pharmacologic Effects of Ci-581, a New Dissociative Anesthetic, in Man. Clin Pharmacol Ther 1965, 6:279-291, doi: 10.1002/cpt196563279

7. Corssen G, Domino EF. Dissociative anesthesia: further pharmacologic studies and first clinical experience with the phencyclidine derivative Cl-581. Anesth Analg 1966, 45:29-40, PMID: 5325977

8. Brewer CL, Davidson JR, Hereward S. Ketamine ("Ketalar"): a safer anaesthetic for ECT. Br J Psychiatry 1972, 120:679-680, doi: 10.1192/ bjp.120.559.679

9. Krystal JH, Karper LP, Seibyl JP, Freeman GK, Delaney R, Bremner JD et al. Subanesthetic effects of the noncompetitive NMDA antagonist, ketamine, in humans. Psychotomimetic, perceptual, cognitive, and neuroendocrine responses. Arch Gen Psychiatry 1994, 51:199-214, doi: 10.1001/archpsyc.1994.03950030035004 
10. Mclnnes EJ, James NM. A comparison of ketamine and methohexital in electroconvulsive therapy. Med J Aust 1972, 1:1031-1032, doi: 10.5694/j.1326-5377.1972.tb108067.x

11. American Psychiatric Association. Task Force on Electroconvulsive T. The Practice of ECT: Recommendations for Treatment, Training and Privileging. Convuls Ther 1990, 6:85-120, PMID: 11659302

12. Krystal AD, Weiner RD, Dean MD, Lindahl VH, Tramontozzi LA 3rd, Falcone $\mathrm{G}$ et al. Comparison of seizure duration, ictal EEG, and cognitive effects of ketamine and methohexital anesthesia with ECT. J Neuropsychiatry Clin Neurosci 2003, 15:27-34, doi: 10.1176/jnp.15.1.27

13. Rasmussen KG, Jarvis MR, Zorumski CF. Ketamine anesthesia in electroconvulsive therapy. Convuls Ther 1996, 12:217-223, PMID: 9034696

14. McDaniel WW, Sahota AK, Vyas BV, Laguerta N, Hategan L, Oswald J. Ketamine appears associated with better word recall than etomidate after a course of 6 electroconvulsive therapies. J ECT 2006, 22:103-106, doi: 10.1097/00124509-200606000-00005

15. Sackeim H, Decina P, Prohovnik I, Malitz S. Seizure threshold in electroconvulsive therapy. Effects of sex, age, electrode placement, and number of treatments. Arch Gen Psychiatry 1987, 44:355-360, doi: 10.1001/archpsyc.1987.01800160067009

16. Berman RM, Cappiello A, Anand A, Oren DA, Heninger GR, Charney DS et al. Antidepressant effects of ketamine in depressed patients. Biol Psychiatry 2000, 47:351-354, doi: 10.1016/s0006-3223(99)00230-9

17. Zarate CA, Jr., Singh JB, Carlson PJ, Brutsche NE, Ameli R, Luckenbaugh $\mathrm{DA}$ et al. A randomized trial of an N-methyl-D-aspartate antagonist in treatment-resistant major depression. Arch Gen Psychiatry 2006, 63:856-864, doi: 10.1001/archpsyc.63.8.856

18. DiazGranados N, Ibrahim LA, Brutsche NE, Ameli R, Henter ID, Luckenbaugh DA et al. Rapid resolution of suicidal ideation after a single infusion of an N-methyl-D-aspartate antagonist in patients with treatment-resistant major depressive disorder. J Clin Psychiatry 2010, 71:1605-1611, doi: 10.4088/JCP.09m05327blu

19. Zarate CA, Jr., Brutsche NE, Ibrahim L, Franco-Chaves J, Diazgranados $\mathrm{N}$, Cravchik A et al. Replication of ketamine's antidepressant efficacy in bipolar depression: a randomized controlled add-on trial. Biol Psychiatry 2012, 71:939-946, doi: 10.1016/j.biopsych.2011.12.010

20. McGirr A, Berlim MT, Bond DJ, Neufeld NH, Chan PY, Yatham LN et al. A systematic review and meta-analysis of randomized controlled trials of adjunctive ketamine in electroconvulsive therapy: efficacy and tolerability. J Psychiatr Res 2015, 62:23-30, doi: 10.1016/j.jpsychires. 2015.01 .003

21. Li DJ, Wang FC, Chu CS, Chen TY, Tang CH, Yang WC et al. Significant treatment effect of add-on ketamine anesthesia in electroconvulsive therapy in depressive patients: A meta-analysis. Eur Neuropsychopharmacol 2017, 27:29-41, doi: 10.1016/j.euroneuro.2016.11.008

22. McGirr A, Berlim MT, Bond DJ, Chan PY, Yatham LN, Lam RW. Adjunctive ketamine in electroconvulsive therapy: updated systematic review and meta-analysis. Br J Psychiatry 2017, 210:403-407, doi: 10.1192/bjp. bp. 116.195826

23. Ren L, Deng J, Min S, Peng L, Chen Q. Ketamine in electroconvulsive therapy for depressive disorder: A systematic review and meta-analysis. J Psychiatr Res 2018, 104:144-156, doi: 10.1016/j.jpsychires.2018.07.003

24. Zheng W, Li XH, Zhu XM, Cai DB, Yang XH, Ungvari GS et al. Adjunctive ketamine and electroconvulsive therapy for major depressive disorder: A meta-analysis of randomized controlled trials. J Affect Disord 2019, 250:123-131, doi: 10.1016/j.jad.2019.02.044

25. Ainsworth NJ, Sepehry AA, Vila-Rodriguez F. Effects of Ketamine Anesthesia on Efficacy, Tolerability, Seizure Response, and Neurocognitive Outcomes in Electroconvulsive Therapy: A Comprehensive Meta-analysis of Double-Blind Randomized Controlled Trials. J ECT 2020, 36:94-105, doi: 10.1097/YCT.0000000000000632
26. Romeo B, Choucha W, Fossati P, Rotge JY. Time-Course of Depression Improvement With Ketamine Adjunction in Electroconvulsive Therapy. J ECT 2016, 32:80-81, doi:10.1097/YCT.0000000000000293

27. Wang X, Chen Y, Zhou X, Liu F, Zhang T, Zhang C. Effects of propofol and ketamine as combined anesthesia for electroconvulsive therapy in patients with depressive disorder. J ECT 2012, 28:128-132, doi: 10.1097/YCT.0b013e31824d1d02

28. Abdallah CG, Fasula M, Kelmendi B, Sanacora G, Ostroff R. Rapid antidepressant effect of ketamine in the electroconvulsive therapy setting. J ECT 2012, 28:157-161, doi: 10.1097/YCT.0b013e31824f8296

29. Loo CK, Katalinic N, Garfield JB, Sainsbury K, Hadzi-Pavlovic D, MacPherson R. Neuropsychological and mood effects of ketamine in electroconvulsive therapy: a randomised controlled trial. J Affect Disord 2012, 142:233-240, doi: 10.1016/j.jad.2012.04.032

30. Jarventausta K, Chrapek W, Kampman O, Tuohimaa K, Bjorkqvist M, Hakkinen $\mathrm{H}$ et al. Effects of S-ketamine as an anesthetic adjuvant to propofol on treatment response to electroconvulsive therapy in treatment-resistant depression: a randomized pilot study. J ECT 2013, 29:158-161, doi: 10.1097/YCT.0b013e318283b7e9

31. Yoosefi A, Sepehri AS, Kargar M, Akhondzadeh S, Sadeghi M, Rafei A et al. Comparing effects of ketamine and thiopental administration during electroconvulsive therapy in patients with major depressive disorder: a randomized, double-blind study. J ECT 2014, 30:15-21, doi: 10.1097/YCT.0b013e3182a4b4c6

32. Rasmussen KG, Ritter MJ. Some considerations of the tolerability of ketamine for ECT anesthesia: a case series and review of the literature. J ECT 2014, 30:283-286, doi:10.1097/YCT.0000000000000100

33. Shams Alizadeh N, Maroufi A, Nasseri K, Sadeghi Najafabadi SH, Mousavi Taghiabad A, Gharibi $F$ et al. Antidepressant Effect of Combined Ketamine and Electroconvulsive Therapy on Patients With Major Depressive Disorder: A Randomized Trial. Iran J Psychiatry Behav Sci 2015, 9:e1578, doi: 10.17795/ijpbs-1578

34. Anderson IM, Blamire A, Branton T, Clark R, Downey D, Dunn G et al. Ketamine augmentation of electroconvulsive therapy to improve neuropsychological and clinical outcomes in depression (KetamineECT): a multicentre, double-blind, randomised, parallel-group, superiority trial. Lancet Psychiatry 2017, 4:365-377, doi: 10.1016/S2215-0366 (17)30077-9

35. Chen Q, Min S, Hao X, Peng L, Meng H, Luo Q et al. Effect of Low Dose of Ketamine on Learning Memory Function in Patients Undergoing Electroconvulsive Therapy-A Randomized, Double-Blind, Controlled Clinical Study. J ECT 2017, 33:89-95, doi: 10.1097/YCT.0000000 000000365

36. Fernie G, Currie J, Perrin JS, Stewart CA, Anderson V, Bennett DM et al. Ketamine as the anaesthetic for electroconvulsive therapy: the KANECT randomised controlled trial. Br J Psychiatry 2017, 210:422-428, doi: 10.1192/bjp.bp.116.189134

37. Kuscu OO, Karacaer F, Biricik E, Gulec E, Tamam L, Gunes Y. Effect of Ketamine, Thiopental and Ketamine-Thiopental Combination during Electroconvulsive Therapy for Depression. Turk J Anaesthesiol Reanim 2015, 43:313-317, doi: 10.5152/TJAR.2015.92668

38. Ray-Griffith SL, Eads LA, Han X, Golden K, Stowe ZN. A Randomized Pilot Study Comparing Ketamine and Methohexital Anesthesia for Electroconvulsive Therapy in Patients With Depression. J ECT 2017, 33:268-271, doi: 10.1097/YCT.0000000000000413

39. Rybakowski JK, Bodnar A, Krzywotulski M, Chlopocka-Wozniak M, Michalak M, Rosada-Kurasinska J et al. Ketamine Anesthesia, Efficacy of Electroconvulsive Therapy, and Cognitive Functions in Treatment-Resistant Depression. J ECT 2016, 32:164-168, doi: 10.1097/ YCT.0000000000000317

40. Salehi B, Mohammadbeigi A, Kamali AR, Taheri-Nejad MR, Moshiri I. Impact comparison of ketamine and sodium thiopental on anesthesia during electroconvulsive therapy in major depression patients with 
drug-resistant; a double-blind randomized clinical trial. Ann Card Anaesth 2015, 18:486-490, doi: 10.4103/0971-9784.166444

41. Zhang M, Rosenheck R, Lin X, Li Q, Zhou Y, Xiao Y et al. A randomized clinical trial of adjunctive ketamine anesthesia in electro-convulsive therapy for depression. J Affect Disord 2018, 227:372-378, doi: 10.1016/j.jad.2017.11.034

42. Zhong $X$, He H, Zhang C, Wang Z, Jiang M, Li Q et al. Mood and neuropsychological effects of different doses of ketamine in electroconvulsive therapy for treatment-resistant depression. J Affect Disord 2016, 201:124-130, doi: 10.1016/j.jad.2016.05.011

43. Carspecken CW, Borisovskaya A, Lan ST, Heller K, Buchholz J, Ruskin $D$ et al. Ketamine Anesthesia Does Not Improve Depression Scores in Electroconvulsive Therapy: A Randomized Clinical Trial. J Neurosurg Anesthesiol 2018, 30:305-313, doi:10.1097/ANA.0000000000000511

44. Erdil F, Begec Z, Kayhan GE, Yologlu S, Ersoy MO, Durmus M. Effects of sevoflurane or ketamine on the QTc interval during electroconvulsive therapy. J Anesth 2015, 29:180-185, doi: 10.1007/s00540-014-1899-2

45. Erdil F, Ozgul U, Colak C, Cumurcu B, Durmus M. Effect of the Addition of Ketamine to Sevoflurane Anesthesia on Seizure Duration in Electroconvulsive Therapy. J ECT 2015, 31:182-185, doi: 10.1097/ YCT.0000000000000225

46. Gamble JJ, Bi H, Bowen R, Weisgerber G, Sanjanwala R, Prasad R et al. Ketamine-based anesthesia improves electroconvulsive therapy outcomes: a randomized-controlled study. Can J Anaesth 2018, 65:636-646, doi: 10.1007/s12630-018-1088-0
47. Erdogan Kayhan G, Yucel A, Colak YZ, Ozgul U, Yologlu S, Karlidag $\mathrm{R}$ et al. Ketofol (mixture of ketamine and propofol) administration in electroconvulsive therapy. Anaesth Intensive Care 2012, 40:305-310, doi: 10.1177/0310057X1204000214

48. Yalcin S, Aydogan H, Selek S, Kucuk A, Yuce HH, Karababa F et al. Ketofol in electroconvulsive therapy anesthesia: two stones for one bird. J Anesth 2012, 26:562-567, doi: 10.1007/s00540-012-1378-6

49. Li XM, Shi ZM, Wang PJ, Hu H. Effects of ketamine in electroconvulsive therapy for major depressive disorder: meta-analysis of randomised controlled trials. Gen Psychiatr 2020, 33:e100117, doi: 10.1136/ gpsych-2019-100117

50. Brunelin J, Iceta S, Plaze M, Gaillard R, Simon L, Suaud-Chagny MF et al. The Combination of Propofol and Ketamine Does Not Enhance Clinical Responses to Electroconvulsive Therapy in Major DepressionThe Results From the KEOpS Study. Front Pharmacol 2020, 11:562137, doi: 10.3389/fphar.2020.562137

51. Zou L, Min S, Chen Q, Li X, Ren L. Subanesthetic dose of ketamine for the antidepressant effects and the associated cognitive impairments of electroconvulsive therapy in elderly patients-A randomized, double-blind, controlled clinical study. Brain Behav 2021, 11:e01775, doi: 10.1002/brb3.1775

52. Chen Q, Dong J, Luo J, Ren L, Min S, Hao X et al. Effects of Low-Dose Ketamine on the Antidepressant Efficacy and Suicidal Ideations in Patients Undergoing Electroconvulsive Therapy. J ECT 2020, 36:25-30, doi: 10.1097/YCT.0000000000000636 


\title{
Electroconvulsive therapy in treatment resistant depression: What is new?
}

\author{
Styliani C. Kaliora \\ 1st Psychiatric Department, University of Athens, Eginition Hospital, Athens, Greece
}

\begin{abstract}
Despite major advances in the treatment of mood disorders, major depression, a common mental disorder, remains a serious public health problem. Electroconvulsive therapy (ECT) regardless of the anesthetic agent used, is the most effective form of treatment in major depression and the gold standard therapy in treatment resistant depression. Ketamine is one of the anesthetic drugs approved by the American Psychiatric Association Task Force Report for use in ECT. However, it has been used infrequently as an anesthetic in ECT. The initial reports suggested that ketamine has antidepressant properties resulting in rapid antidepressant response when administered in subanesthetic dose $(0.5 \mathrm{mg} / \mathrm{kg})$ in slow intravenous injection in patients suffering from depression. In recent trials has been reported that ketamine as the only anesthetic or as an adjunctive to another anesthetic agent may enhance the antidepressant effect of ECT either by increasing efficacy or by producing a rapid antidepressant response. ECT with ketamine may also cause less cognitive side effects. The most notable limitations of these studies are the small number of patients enrolled and several methodological differences (patients characteristics, electrode placements, titration method, anesthetic agent used with ketamine). The results of the clinical trials have been summarized in six meta-analysis and suggest that ketamine when used as a sole anesthetic agent or as an adjunctive anesthetic in ECT may accelerates the antidepressant response but does not augment ECT efficacy. It also does not improve the cognitive profile of the treatment. Larger, double-blind randomized controlled trial are needed for a definite conclusion.
\end{abstract}

KEYWORDS: Electroconvulsive therapy, ketamine, depression, safety, cognitive functions. 